\title{
氧氯化锑的形貌转变: 从束状到准圆形
}

\author{
周静 ${ }^{\mathbb{1} 2}$ ，赵赫威 ${ }^{(1)}$, 李丽东 ${ }^{(1)}$, 郭林 ${ }^{(1 *}$ \\ (1) 北京航空航天大学化学与环境学院, 北京 100191; \\ (2) 东北电力大学化学工程学院, 吉林 132012 \\ * 联系人, E-mail: guolin@buaa.edu.cn
}

2011-05-17 收稿, 2011-05-24 接受

国家重点基础研究发展计划(2010CB93470)和国家自然科学基金(50725208, 50923019)资助项目

\begin{abstract}
摘要 在室温下的溶液中以温和易行的实验条件, 成功制备了各种形貌不同的氯氧化锑, 包括 束状、菱形片状、类似树叶的椭圆状和准圆片状. 利用 $\mathrm{X}$ 射线衍射(XRD), 扫描电子显微镜 (SEM)和透射电子显微镜(TEM) 等手段对制备的各种不同形貌和结构的样品进行了表征. 在此 基础上, 提出了可能的形成机理.
\end{abstract}

\section{关键词}

氯氧化锑

束状

准圆片

晶体分裂

形貌转变
过去 10 多年, 合成精确可控形貌和尺寸的无机 材料是材料科学领域的一个研究热点. 材料在光学、 电学、磁学以及催化方面许多独特的性能不仅依赖于 它们的成分, 同时与其结构、晶相、形貌、尺寸及尺 寸分布密不可分 ${ }^{[1 \sim 8]}$. 近年来, 形貌及尺寸可控的无 机纳米材料受到了人们的广泛关注. 尤其是那些具 有预期形貌、晶相及尺寸的材料在不同领域有着许多 潜在的应用前景, 得到了研究人员的极大关注.

在 $\mathrm{Sb}$ 系阻燃材料中, 氯氧化锑 $\left(\mathrm{SbOCl}, \mathrm{Sb}_{4} \mathrm{O}_{5} \mathrm{Cl}_{2}\right.$, $\mathrm{Sb}_{8} \mathrm{O}_{11} \mathrm{Cl}_{2}$ ) 具有优良的阻燃特性, 既可单独作为阻燃 剂, 也可以与卤素配合, 发挥阻燃协效的作用 ${ }^{[9]}$. 目 前, 采用温和简单的溶液法制备氯氧化锑纳米材料 的报道还相对较少. 研究者采用溶液法和水热法合 成了 $\mathrm{Sb}_{4} \mathrm{O}_{5} \mathrm{Cl}_{2}$ 纳米带 ${ }^{[10]}$, 不同形貌的 $\mathrm{Sb}_{8} \mathrm{O}_{11} \mathrm{Cl}_{2}$ 晶体, 如纳米带 ${ }^{[10]}$ 、纳米线、纳米花、纳米线束、纳米带 束、纳米片束及空心棱柱 ${ }^{[11]}$, 以及针状的 $\mathrm{Sb}_{8} \mathrm{O}_{11} \mathrm{Cl}_{2}$ $\left(\mathrm{H}_{2} \mathrm{O}\right)_{6}$ 微晶 ${ }^{[12]}$. 在目前的合成方法中, 仍存在着产 物形貌不均匀以及混相等问题.

本文报道了一种在室温下用溶液法合成不同形 貌 $\mathrm{Sb}_{8} \mathrm{O}_{11} \mathrm{Cl}_{2}\left(\mathrm{H}_{2} \mathrm{O}\right)_{6}$ 的简单方法. 通过改变 $\mathrm{NaOH}$ 的 浓度, 从而改变产物晶体的分裂程度, 实现了最终产 物氯氧化锑的形貌从束状、菱形片状、椭圆叶片状,
最后转变成准圆片状的衍变, 并详细分析了相应形 貌的转变机理.

\section{1 实验}

(i ) 制备方法. 本实验所用的试剂都是分析纯. 首先, 分别将 $0.5550 \mathrm{~g}$ 聚乙烯吡咯烷酮 $\left(\mathrm{PVP}, M_{\mathrm{W}}\right.$ 58000 )和 $30 \mathrm{~mL} 0.033 \mathrm{~mol} / \mathrm{L} \mathrm{SbCl}{ }_{3}$ 乙二醇 $(\mathrm{EG})$ 溶液加 人圆底烧瓶中，持续搅拌一段时间后形成无色透明 的溶液, 然后再往其中逐滴加人不同浓度的 $\mathrm{NaOH}$ 溶液 $(0.25,0.35,0.40,0.45,0.48$ 和 $0.50 \mathrm{~mol} / \mathrm{L})$, 连续 搅拌 $5 \mathrm{~h}$, 将上述溶液静置 $4 \mathrm{~h}$ 后得到白色的沉淀. 利 用乙醇多次洗涤样品, 最后将之置于 $50^{\circ} \mathrm{C}$ 的烘箱内 干燥 $12 \mathrm{~h}$ 得到白色粉末。

(ii) 实验仪器. 用 $\mathrm{X}$ 射线衍射仪 (XRD)、场发 射扫描电子显微镜(FESEM)及场发射透射电子显微 镜(FTEM)对所制备的样品进物相、形貌及微结构进 行表征. $X$ 射线衍射仪为日本理学公司 Rigaku Dmac 2200 型, 铜靶 $(\lambda=1.5416 \AA)$, 扫描速度为 $0.02 \%$, 扫描范围为 $10^{\circ} \sim 60^{\circ}$. 场发射扫描电子显微镜为日本 日立公司 Hitachi S-4800 型, 加速电压为 $5 \mathrm{kV}$. 透射 电子显微镜为日本电子公司 JEOL JEM-2100F 型, 加速电压为 $200 \mathrm{kV}$. 利用美国康塔公司 NOVA 2200e

英文版见: Zhou J, Zhao H W, Li L D, et al. Shape evolution of antimony oxychloride from sheaf-like to quasi-wafer structures. Chinese Sci Bull, 2011, 56, doi: 10.1007/s11434-011-4735-1 
型比表面孔径分析仪, 在 $77 \mathrm{~K}$ 的条件下测试了产物 的比表面积, 采用 BET 法计算比表面积, 而孔容及 孔分布则采用 $\mathrm{BJH}$ 方程由脱附曲线进行计算.

\section{2 结果与讨论}

\section{1 样品的表征}

$\mathrm{X}$ 射线衍射用来表征样品的化学结构以及物相. 如图 1 所示, 所有样品的衍射峰都能与斜方晶系的 $\mathrm{Sb}_{8} \mathrm{O}_{11} \mathrm{Cl}_{2}\left(\mathrm{H}_{2} \mathrm{O}\right)_{6}$ (JCPDS 卡片, 编号 77-1584) 相对 应, 其晶格常数为 $a=0.9618 \mathrm{~nm}, b=1.314 \mathrm{~nm}$ 和 $c=0.4078 \mathrm{~nm}$. 产物 $\mathrm{X}$ 射线衍射峰的强度与标准样品 $\mathrm{XRD}$ 谱图进行对比, 可以用来讨论晶体的生长取

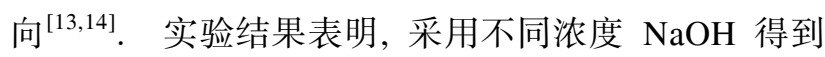
的不同形貌产物的 XRD 谱图中, (020)与(031)衍射峰 之间的强度比值存在明显变化. 在图 1(a)中, (020)与 (031)衍射峰之间的强度比值相比于标准 XRD 图谱中 所对应的强度比值略低 (分别是 0.57 和 0.75). 而从 图 1(b) (e)观察, 随着 $\mathrm{NaOH}$ 浓度的增加, XRD 谱图 中(020)与(031)衍射峰之间的强度比值是逐渐增加的 (对应的数值分别是 $0.62,0.92,1.01$ 和 3.57). 另外, 在图 1(e)中, (040) 与(031), (060) 与(031) 衍射峰之 间的强度比值较其他谱图中对应的强度比值也明显 增加了. 这些结果都表明在生长过程中, 晶体具有各 向异性的生长, 我们将在后续的讨论中进行论证.

图 2 是采用不同 $\mathrm{NaOH}$ 浓度得到的产物的 SEM 图. 如图 2(a) 和(b)所示, 当 $\mathrm{NaOH}$ 浓度为 0.25 或 0.35 $\mathrm{mol} / \mathrm{L}$ 时, 产物皆为束状的氯氧化锑. 尽管两者都是 所谓的“束状”结构, 顶部和底端都呈扇形发散, 中间

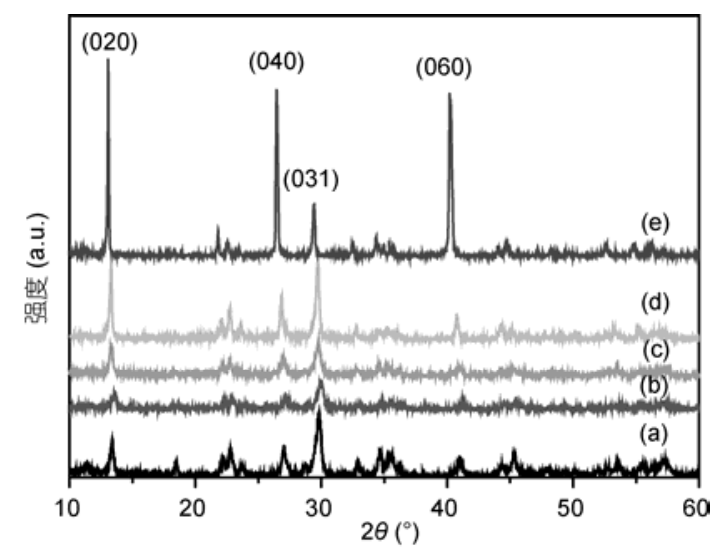

图 1 不同 $\mathrm{NaOH}$ 浓度下得到样品的 XRD 谱图 (a) 0.25 , (b) 0.35 , (c) 0.40 , (d) 0.45 和 (e) $0.50 \mathrm{~mol} / \mathrm{L}$
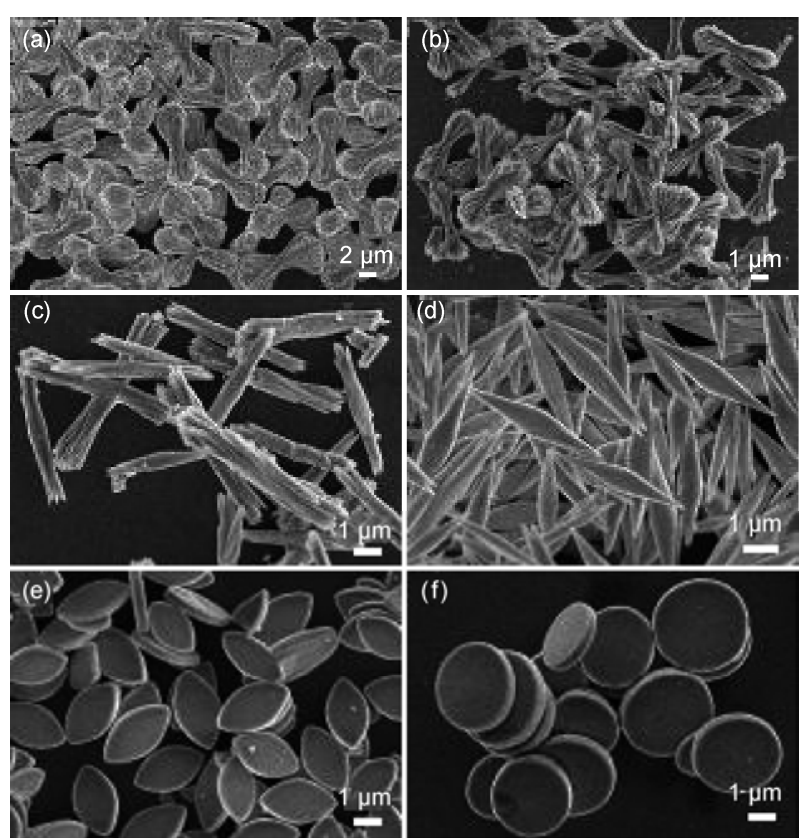

图 2 不同 $\mathrm{NaOH}$ 浓度下得到的 $\mathrm{Sb}_{8} \mathrm{O}_{11} \mathrm{Cl}_{2}\left(\mathrm{H}_{2} \mathrm{O}\right)_{6}$ 样品 $\mathrm{SEM}$ 图 (a) 0.25 , (b) 0.35 , (c) 0.40 , (d) 0.45 , (e) 0.48 和(f) $0.50 \mathrm{~mol} / \mathrm{L}$

则紧密地捆绑在一起, 但产物 (a)向外扩张的程度明 显大于产物(b)，而且其长度也长于后者. 当 $\mathrm{NaOH}$ 浓 度增加至 $0.40 \mathrm{~mol} / \mathrm{L}$ 时, 产物的形貌是束状向菱形片 状过渡的中间结构, 主要为准菱形状, 两端仍有分叉, 如图 2(c)所示. 继续增加 $\mathrm{NaOH}$ 浓度至 $0.45 \mathrm{~mol} / \mathrm{L}$, 会形成大多数呈菱形片状结构的产物, 极少数菱形 片的两端还存在少量分叉(图 4(d)). 将 $\mathrm{NaOH}$ 浓度提 高至 $0.48 \mathrm{~mol} / \mathrm{L}$ 时得到如图 2(e)所示的椭圆叶片状 结构, 其长度约为 $3 \mu \mathrm{m}$, 宽约为 $1.8 \mu \mathrm{m}$, 厚度约为 $0.4 \mu \mathrm{m}$. 将 $\mathrm{NaOH}$ 浓度继续升至 $0.50 \mathrm{~mol} / \mathrm{L}$ 时, 所得 产物为准圆片状的结构, 如图 2(f)所示. 如果 $\mathrm{NaOH}$ 浓度超过 $0.50 \mathrm{~mol} / \mathrm{L}$, 得到的产物则是 $\mathrm{Sb}_{2} \mathrm{O}_{3}$ 而不是 $\mathrm{Sb}_{8} \mathrm{O}_{11} \mathrm{Cl}_{2}\left(\mathrm{H}_{2} \mathrm{O}\right)_{6}$, 这在我们其他的研究中进行了讨 论 ${ }^{[15]}$. 图 2 清晰地呈现出通过改变 $\mathrm{NaOH}$ 浓度, 产物 的形貌由束状、扩张程度减小的束状向准圆片状的整 个转变过程.

图 3 所示为所得样品的 TEM 形貌表征结果. 图 3 更加清晰地呈现出产物的形貌由束状、菱形片 状、椭圆叶片状到准圆片状的转变. 图 3(a) (f)的插 图为每种产物顶端的选区电子衍射(SAED)谱图. 在 图 3(a)和(b)的插图中, SAED 谱图呈现的是由断续亮 的延长点组成的衍射环, 表明束状微晶是一种多晶 结构. 而图 3(c)到(f)中的 SAED 谱图呈现规则的点 


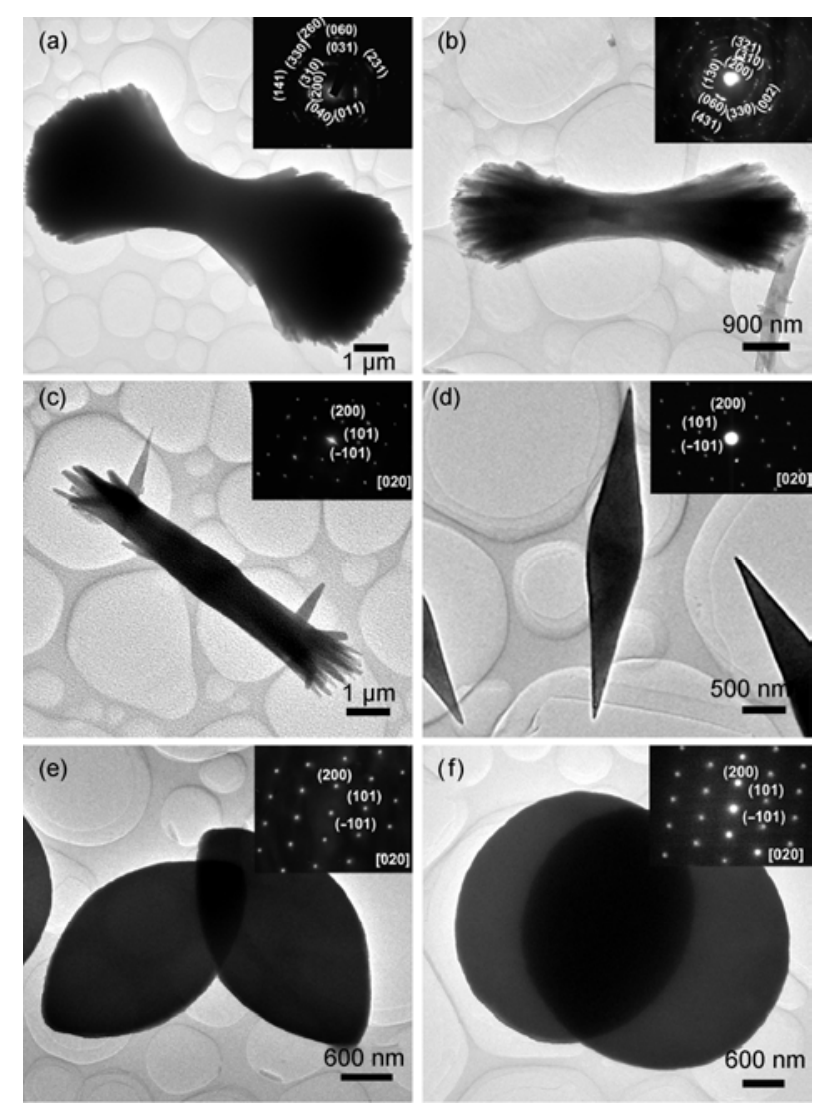

图 3 不同 $\mathrm{NaOH}$ 浓度下得到的 $\mathrm{Sb}_{8} \mathrm{O}_{11} \mathrm{Cl}_{2}\left(\mathrm{H}_{2} \mathrm{O}\right)_{6}$ 样品 $\mathrm{TEM}$ 图 (a) 0.25 , (b) 0.35 , (c) 0.40 , (d) 0.45 , (e) 0.48 和 (f) $0.50 \mathrm{~mol} / \mathrm{L}$. 插图是相应的选区电子衍射(SAED)谱图

阵结构, 表明所得到的产物是单晶, 对应于单斜晶系 的 $\mathrm{Sb}_{8} \mathrm{O}_{11} \mathrm{Cl}_{2}\left(\mathrm{H}_{2} \mathrm{O}\right)_{6}$ 的 [020]晶面, 这与之前 XRD 的结 果一致，表明产物形貌从束状到准圆片状的生长是 有一定取向的.

图 4(a) (c)为 $\mathrm{Sb}_{8} \mathrm{O}_{11} \mathrm{Cl}_{2}\left(\mathrm{H}_{2} \mathrm{O}\right)_{6}$ 的氮等温吸附-脱 附曲线, 表现为典型的 $\mathrm{IV}$ 吸附等温线. 采用不同 $\mathrm{NaOH}$ 浓度(分别是 $0.25,0.40$ 和 $0.45 \mathrm{~mol} / \mathrm{L}$ ) 得到的 $\mathrm{Sb}_{8} \mathrm{O}_{11} \mathrm{Cl}_{2}\left(\mathrm{H}_{2} \mathrm{O}\right)_{6}$ 样品的 BET 表面积分别是 $9.49 \pm 0.5$, $8.98 \pm 0.5$ 和 $8.47 \pm 0.5 \mathrm{~m}^{2} / \mathrm{g}$. 随着 $\mathrm{NaOH}$ 浓度的增加, 产物的 BET 表面积有所降低. 为了进一步研究不同 产物的孔结构, 我们采用 BJH 方程计算得到了孔分布 曲线, 如图 4(a) (c)插图所示. 而不同 $\mathrm{NaOH}$ 浓度得到 的 $\mathrm{Sb}_{8} \mathrm{O}_{11} \mathrm{Cl}_{2}\left(\mathrm{H}_{2} \mathrm{O}\right)_{6}$ 产物孔径大小的中心值分别为 3.83 , 3.06 和 $2.75 \mathrm{~nm}$, 这意味着产物具有微孔结构.

\section{2 生长机理}

针对之前报道过的其他束状晶体, 包括 $\mathrm{Bi}_{2} \mathrm{~S}_{3}{ }^{[16 \sim 18]}$,
$\beta-\mathrm{FeO}(\mathrm{OH})^{[19]}, \mathrm{BaWO}_{4}{ }^{[20,21]}$, 镧矾酸盐 ${ }^{[22]}, \mathrm{Sb}_{2} \mathrm{~S}_{3}$ 与 $\mathrm{Sb}_{2} \mathrm{Se}_{3}{ }^{[23,24]}, \mathrm{CuO}^{[25,26]}, \mathrm{CeO}_{2}{ }^{[27]}$ 与掺杂 $\mathrm{Tb}$ 的 $\mathrm{CePO}_{4}{ }^{[28]}$, 当前广泛认为这种束状结构形成的机理是由于在生 长过程中存在晶体分裂. 溶液的过饱和或加人一些 影响晶体成长过程的添加剂都可能导致晶体分裂. 实验结果表明, 在本文的反应体系中 PVP 的加人对 束状结构的形成是至关重要的. 在相同条件下，如果 不添加 PVP 仅能得到有一些分叉的不规则的纳米棒 (图 4(a)). 而 PVP 作为一种有效的晶面掩蔽剂和表面 稳定剂, 可能会选择性地吸附到特定的晶面上, 从而 控制晶面的生长速率. 在本溶液体系中, PVP 选择吸 附在特定的晶面上, 使某个晶面优先快速生长, 最终 导致晶体分裂.

PVP 的用量对产物的形貌有很大的影响. 图 5 是 在没有 PVP 存在下, 采用不同 $\mathrm{NaOH}$ 浓度所得的 $\mathrm{Sb}_{8} \mathrm{O}_{11} \mathrm{Cl}_{2}\left(\mathrm{H}_{2} \mathrm{O}\right)_{6}$ 样品的 SEM 图. 对比之前的结果, 很明显看到, 当 $\mathrm{NaOH}$ 浓度低于 $0.45 \mathrm{~mol} / \mathrm{L}$ 时, 所得 产物与有 PVP 存在条件下所得产物的形貌是不同的; 而当 $\mathrm{NaOH}$ 浓度增大至 $0.45,0.48$ 或 $0.50 \mathrm{~mol} / \mathrm{L}$ 时, 仍可得到类似的菱形片状、准椭圆片状或准圆片状的 形貌.

我们用晶体分裂理论及晶体成核与生长理论来 解释不同形貌产物的形成原因. 众所周知, 如果溶液 的过饱和超过了一定的临界值, 就可能发生晶体分 裂, 在快速生长的条件下就会形成束状结构. 然而, 当 $\mathrm{NaOH}$ 浓度增加时, 在很短的时间内会形成更多 的晶核, 之后这些大量的晶核缓慢生长, 这将抑制晶 体分裂过程 ${ }^{[18,29]}$. 与之类似, 在添加了 PVP 的实验结 果中, 晶体分裂的程度是随着 $\mathrm{NaOH}$ 浓度的增加 而减小的, 如分裂程度大的束状结构 (所用 $\mathrm{NaOH}$ 浓度为 $0.25 \mathrm{~mol} / \mathrm{L})$ 、程度减少的束状 $(\mathrm{NaOH}$ 浓度为 $0.35 \mathrm{~mol} / \mathrm{L})$ 、分裂程度较小的小束状 $(\mathrm{NaOH}$ 浓度为 $0.40 \mathrm{~mol} / \mathrm{L})$ 、几乎没有分裂的菱形片状 $(\mathrm{NaOH}$ 浓度为 $0.45 \mathrm{~mol} / \mathrm{L}$ ) 以及没有分裂的椭圆叶片和准圆片状结 构 $(\mathrm{NaOH}$ 浓度为分别是 0.48 和 $0.50 \mathrm{~mol} / \mathrm{L})$. 因此, 在 有 PVP 存在的溶液体系中, 所得产物形貌的变化与 反应物的浓度是相关的. 而在没有 PVP 的情况下, 当 $\mathrm{NaOH}$ 浓度低于 $0.45 \mathrm{~mol} / \mathrm{L}$ 时, 所得产物的形貌与 有 PVP 条件下得到的产物形貌是不同的，而 $\mathrm{NaOH}$ 浓度大于 $0.45 \mathrm{~mol} / \mathrm{L}$ 时, 产物的形貌又与有 $\mathrm{PVP}$ 条 件下得到的产物形貌类似. 因此, 我们推测, 当 $\mathrm{NaOH}$ 浓度低于 $0.45 \mathrm{~mol} / \mathrm{L}$ 时, PVP 的存在对于形成 

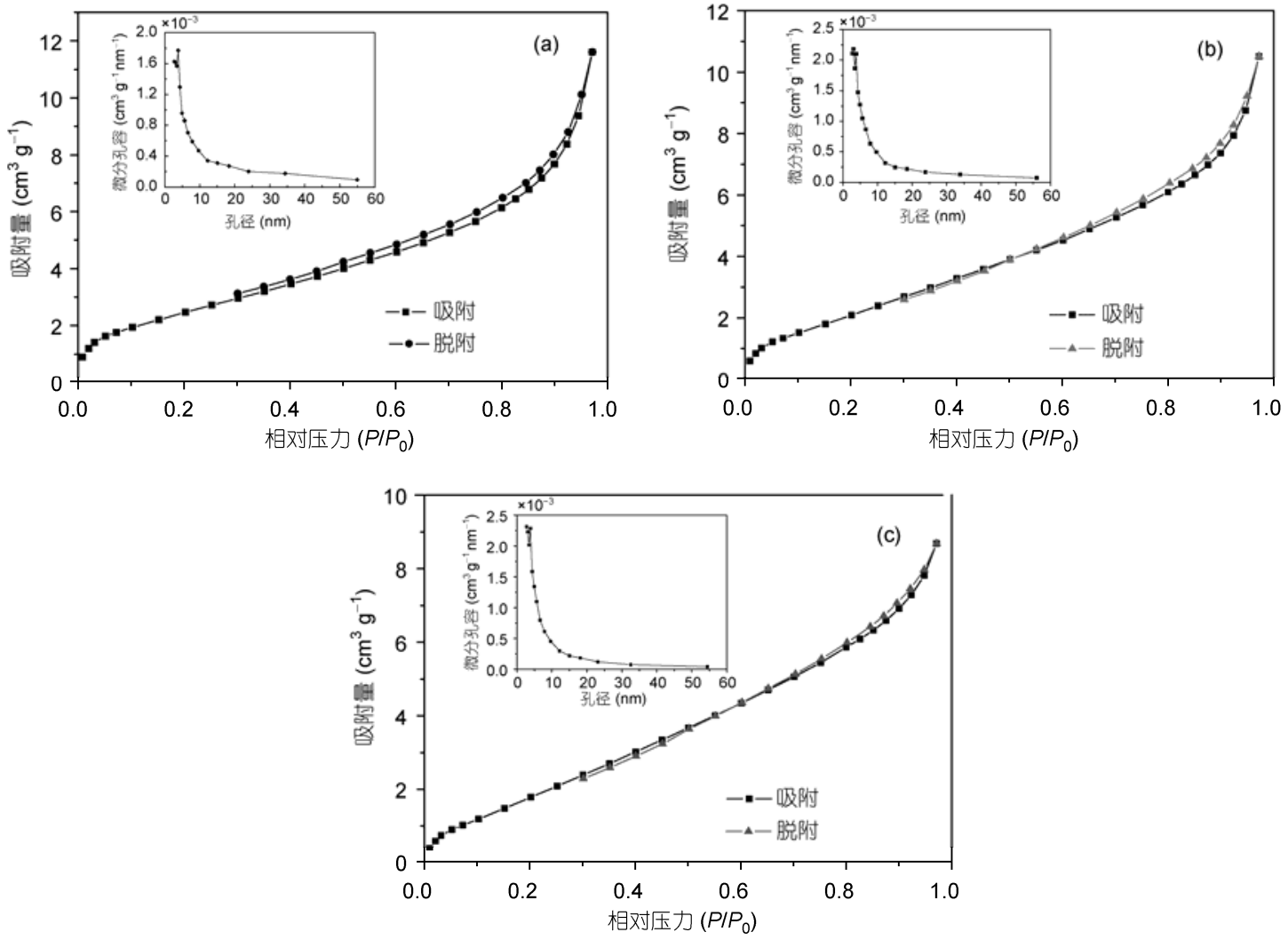

图 4 不同 $\mathrm{NaOH}$ 浓度下得到的 $\mathrm{Sb}_{8} \mathrm{O}_{11} \mathrm{Cl}_{2}\left(\mathrm{H}_{2} \mathrm{O}\right)_{6}$ 氮等温吸附-脱附曲线和孔分布曲线 (a) 0.25 , (b) 0.40 , (c) $0.45 \mathrm{~mol} / \mathrm{L}$

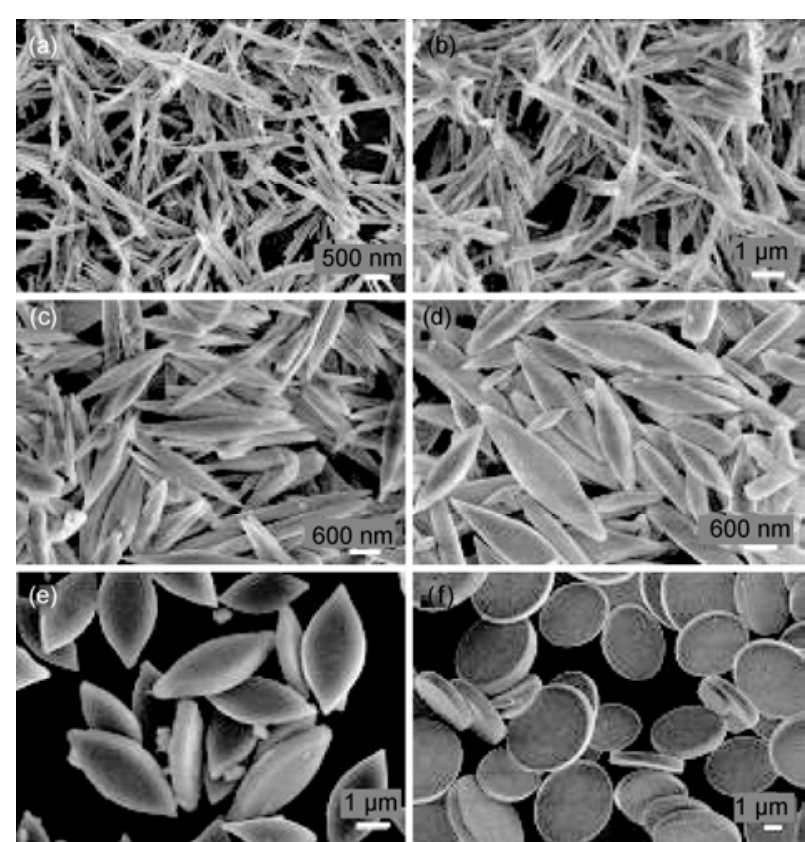

图 5 在没有 PVP 存在下, 不同 $\mathrm{NaOH}$ 浓度所得的 $\mathrm{Sb}_{8} \mathbf{O}_{11} \mathrm{Cl}_{2}\left(\mathrm{H}_{2} \mathrm{O}\right)_{6}$ 样品 SEM 图

(a) 0.25 , (b) 0.35 , (c) 0.40 , (d) 0.45 , (e) 0.48 和 (f) $0.50 \mathrm{~mol} / \mathrm{L}$
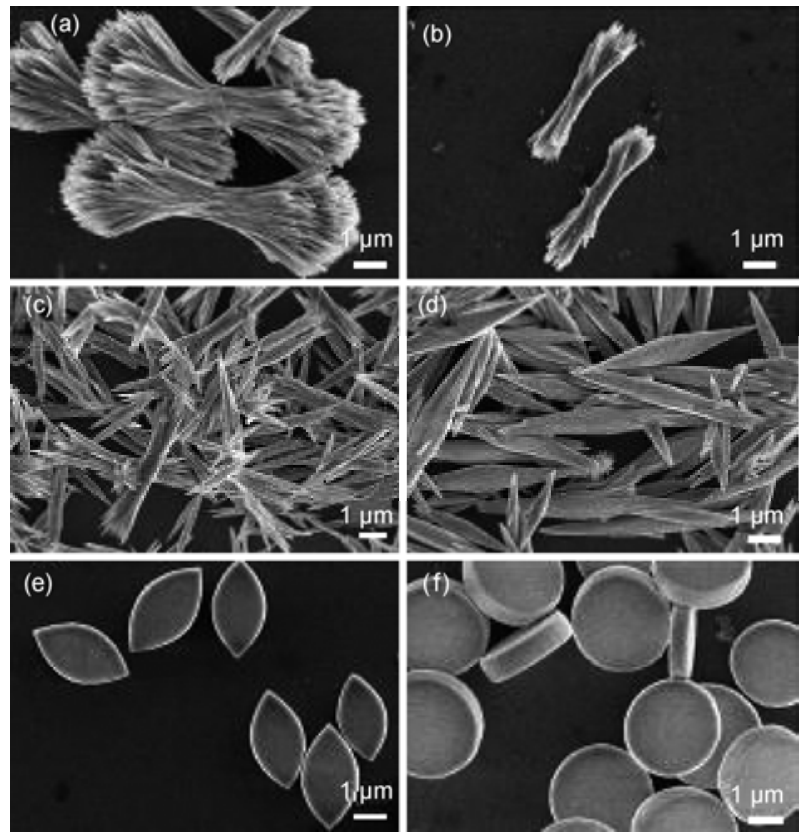

图 6 不同 $\mathbf{S b}^{3+}$ 摩尔量所得到的 $\mathrm{Sb}_{8} \mathbf{O}_{11} \mathrm{Cl}_{2}\left(\mathrm{H}_{2} \mathbf{O}\right)_{6}$ 样品 $\mathrm{SEM}$ 图 (a) 0.90 , (b) 0.80 , (c) 0.70 , (d) 0.60 , (e) 0.53 和(f) $0.50 \mathrm{mmol}$. 所用 的 $\mathrm{NaOH}$ 浓度为 $0.25 \mathrm{~mol} / \mathrm{L}$ 
具有不同晶体分裂程度的束状结构很重要; 而当 $\mathrm{NaOH}$ 浓度大于等于 $0.45 \mathrm{~mol} / \mathrm{L}$ 时, $\mathrm{NaOH}$ 浓度的作 用就显现出来了, 无论是否存在 PVP, 都会在很短的 时间内形成更多的晶核, 然后缓慢生长, 最终导致晶 体没有分裂. 同样这个结论也可以通过固定 $\mathrm{NaOH}$ 浓度, 改变体系中 $\mathrm{Sb}^{3+}$ 的摩尔量来证明(图 6). 从图 6(a) (f) 可清楚地观察到, 随着 $\mathrm{Sb}^{3+}$ 浓度的减少, 产 物的形貌从束状、菱形片状、椭圆叶片状到准圆片状 发生了一系列的转变. 这与之前固定 $\mathrm{Sb}^{3+}$ 的摩尔量, 改变 $\mathrm{NaOH}$ 浓度的结果是一致的.

基于上述研究, 可以推测出体系中存在 PVP 以 及反应物之间的比率, 对于从束状到准圆片状结构 的形貌转变都是十分关键的因素. 图 7 是不同形貌的 $\mathrm{Sb}_{8} \mathrm{O}_{11} \mathrm{Cl}_{2}\left(\mathrm{H}_{2} \mathrm{O}\right)_{6}$ 微晶生长过程的示意图. 当体系中 存在 $\mathrm{PVP}, \mathrm{Sb}^{3+} / \mathrm{OH}^{-}$的比率为 $1: 1.5$ 时, 会发生晶体分 裂，从而形成束状结构的产物. 当 $\mathrm{Sb}^{3+} / \mathrm{OH}^{-}$的比率变 为 $1: 2.1$ 时, 晶体分裂的程度会减小, 形成扩张程度 相对减小的束状结构. 当 $\mathrm{Sb}^{3+} / \mathrm{OH}^{-}$的比率达到 $1: 2.4$ 和 1:2.7 时, 只存在少量的晶体分裂, 所得产物的顶 端只有少量的分叉. 而 $\mathrm{Sb}^{3+} / \mathrm{OH}^{-}$的比率分别为 $1: 2.88$ 和 $1: 3$ 时, 所得到的椭圆叶片状和准圆片状结 构表明晶体在生长过程中没有发生晶体分裂. 在合 成 $\mathrm{Sb}_{8} \mathrm{O}_{11} \mathrm{Cl}_{2}\left(\mathrm{H}_{2} \mathrm{O}\right)_{6}$ 微晶中, 添加 $\mathrm{PVP}$ 和采用不同浓度 的反应物会直接影响生长过程中晶体分裂的程度.

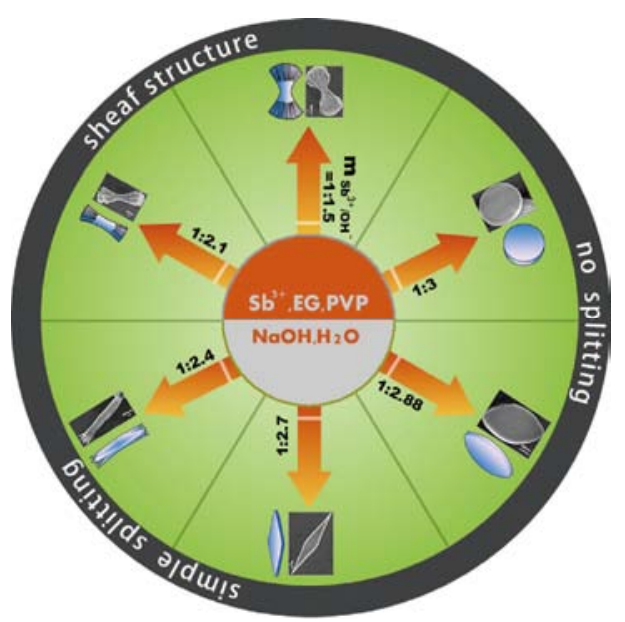

图 7 不同形貌的 $\mathrm{Sb}_{8} \mathrm{O}_{11} \mathrm{Cl}_{2}\left(\mathrm{H}_{2} \mathrm{O}\right)_{6}$ 微晶生长过程的示意图

\section{3 结论}

在有 PVP 存在的条件下, 采用简单温和溶液法 合成了形貌可控的 $\mathrm{Sb}_{8} \mathrm{O}_{11} \mathrm{Cl}_{2}\left(\mathrm{H}_{2} \mathrm{O}\right)_{6}$ 微晶. 仅通过改 变 $\mathrm{NaOH}$ 的浓度就很容易得到一系列不同形貌的产 物，如束状、菱形片状、椭圆叶片状和准圆片状. 基 于推测出的生长机理, 我们相信这种简单易行的方 法对于合成其他形貌可控的无机材料具有潜在的指 导作用. 另外, 这种氯氧化锑作为有前景的阻燃剂, 因其自身包含卤素可单独作为阻燃剂, 也可为其他 卤化的有机化合物提供良好的协效作用.

\section{参考文献}

1 Min Y, Akbulut M, Kristiansen K, et al. The role of interparticle and external forces in nanoparticle assembly. Nat Mater, 2008, 7: $527-538$

2 Wang D, Lieber C M. Inorganic materials: Nanocrystals branch out. Nat Mater, 2003, 2: 355-356

3 Nguyen T D, Dinh C T, Do T O. Shape- and size-controlled synthesis of monoclinic ErOOH and cubic $\mathrm{Er}_{2} \mathrm{O}_{3}$ from micro- to nanostructures and their upconversion luminescence. ACS Nano, 2010, 4: 2263-2273

4 Jang S Y, Song Y M, Kim H S, et al. Three synthetic routes to single-crystalline PbS nanowires with controlled growth direction and their electrical transport properties. ACS Nano, 2010, 4: 2391-2401

5 Gao J H, Gu H W, Xu B. Multifunctional magnetic nanoparticles: Design, synthesis, and biomedical applications. Acc Chem Res, 2009, 42: 1097-1107

6 Xiong Y J, Wiley B J, Xia Y N. Nanocrystals with unconventional shapes-a class of promising catalysts. Angew Chem Int Ed, 2007, 46: $7157-7159$

7 Lu X B, Wen Z H, Li J H. Hydroxyl-containing antimony oxide bromide nanorods combined with chitosan for biosensors. Biomaterials, 2006, 27: 5740-5747

8 Li J H, Zhang J Z. Optical property and applications of hybrid semiconductor nanomaterials. Coordin Chem Rev, 2009, 253: 3015-3041

9 Costa L, Paganetto G, Bertelli G, et al. Thermal decomposition of antimony oxyhalides: I. Oxychlorides. J Therm Anal, 1990, 36: $1141-1153$

10 Chen X Y, Huh H S, Lee S W. Hydrothermal synthesis of antimony oxychloride and oxide nanocrystals: $\mathrm{Sb}_{4} \mathrm{O}_{5} \mathrm{Cl}_{2}, \mathrm{Sb}_{8} \mathrm{O}_{11} \mathrm{Cl}_{2}$, and $\mathrm{Sb}_{2} \mathrm{O}_{3}$. J Solid State Chem, 2009, 181: 2127-2132 
11 Tang J J, Wang Y, Jiao Z, et al. Self-assembly nanostructures of one-dimensional antimony oxide and oxychloride. Mater Lett, 2009, 63: $1481-1484$

12 Li B J, Zhao Y B, Xu X M, et al. A simple method for the preparation of containing Sb nano- and microcrystallines via an ultrasound agitation. Ultraso Sonochem, 2007, 14: 557-562

13 Sui Y M, Fu W Y, Yang H B, et al. Low temperature synthesis of $\mathrm{Cu}_{2} \mathrm{O}$ crystals: Shape evolution and growth mechanism. Cryst Growth Des, 2010, 10: 99-108

14 Wang H H, Tian F, Li X P, et al. Preparation and shape evolution of cuprous oxide in the solution phases of copper (II) dodecyl sulfate. Powder Technol, 2010, 197: 298-302

15 Zhou J, Zhao H W, Li L D, et al. One-step synthesis and flame retardancy of sheaf-like microcrystal antimony oxychloride. J Nanosci Nanotech, 2011 (in press)

16 Tang J, Alivisatos A P. Crystal splitting in the growth of $\mathrm{Bi}_{2} \mathrm{~S}_{3}$. Nano Lett, 2006, 6: 2701-2706

17 Li L S, Sun N J, Huang Y Y, et al. Topotactic transformation of single-crystalline precursor discs into disc-Like Bi $\mathrm{S}_{3}$ nanorod networks. Adv Funct Mater, 2008, 18: 1194-1201

18 Tian L, Tan H Y, Vittal J J. Morphology-controlled synthesis of $\mathrm{Bi}_{2} \mathrm{~S}_{3}$ nanomaterials via singleand multiple-source approaches. Cryst Growth Des, 2008, 8: 734-738

$19 \mathrm{Hu}$ Y H, Chen K Z. Crystal splitting in the growth of $\beta-\mathrm{FeO}(\mathrm{OH})$. J Cryst Growth, 2007, 308: 185-188

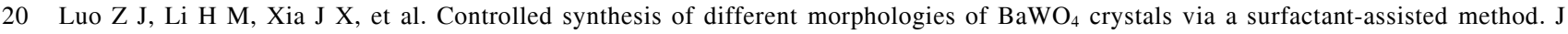
Cryst Growth, 2007, 300: 523-529

21 He J H, Han M, Shen X P, et al. Crystal hierarchically splitting in growth of BaWO in positive cat-anionic microemulsion. J Cryst Growth, 2008, 310: 4581-4586

22 Deng H, Liu C M, Yang S H, et al. Additive-mediated splitting of lanthanide orthovanadate nanocrystals in water: Morphological evolution from Rods to sheaves and to spherulites. Cryst Growth Des, 2008, 8: 4432-4439

23 Chen G Y, Dneg B, Cai G B, et al. The fractal splitting growth of $\mathrm{Sb}_{2} \mathrm{~S}_{3}$ and $\mathrm{Sb}_{2} \mathrm{Se}_{3}$ hierarchical nanostructures. J Phys Chem C, 2008, 112 : 672-679

24 Ota J, Roy P, Srivastava S K, et al. Morphology evolution of $\mathrm{Sb}_{2} \mathrm{~S}_{3}$ under hydrothermal conditions: Flowerlike structure to nanorods. Cryst Growth Des, 2008, 8: 2019-2023

25 Zhang M, Xu X D, Zhang M L. Hydrothermal synthesis of sheaf-like CuO via ionic liquids. Mater Lett, 2008, 62: 385-388

26 Pan Q T, Huang K, Ni S B, et al. Synthesis of sheaf-like CuO from aqueous solution and their application in lithium-ion batteries. J Alloys Comp, 2009, 484: 322-326

27 Kim S, Lee J S, Mitterbauer C, et al. Anomalous electrical conductivity of nanosheaves of CeO 2 . Chem Mater, 2009, 21: 1182-1186

28 Zhang F, Wong S S. Ambient large-scale template-mediated synthesis of high-aspect ratio single-crystalline, chemically doped rare-earth phosphate nanowires for bioimaging. ACS Nano, 2010, 4: 99-112

29 Liu K, You H P, Jia G, et al. Hierarchically nanostructured coordination polymer: Facile and rapid fabrication and tunable morphologies. Cryst Growth Des, 2010, 10: 790-797 